\title{
Live cell imaging by multifocal multiphoton microscopy
}

\author{
Martin Straub $^{\mathrm{a} 1)}$, Peter Lodemann ${ }^{\mathrm{b}}$, Phillip Holroyd ${ }^{\mathrm{b}}$, Reinhard Jahn ${ }^{\mathrm{b}}$, Stefan W. Hell ${ }^{\mathrm{a}}$ \\ a High Resolution Optical Microscopy Group, Max-Planck-Institute for Biophysical Chemistry, Göttingen/Germany \\ b Department of Neurobiology, Max-Planck-Institute for Biophysical Chemistry, Göttingen/Germany
}

Received April 5, 2000

Received in revised version June 15, 2000

Accepted June 20, 2000

Two-photon - multifocal - live cell microscopy - PC12 cells - bovine chromaffin cells

Multifocal multiphoton microscopy (MMM) permits parallel multiphoton excitation by scanning an array of high numerical aperture foci across a plane in the sample. MMM is particularly suitable for live cell investigations since it combines advantages of standard multiphoton microscopy such as optical sectioning and suppression of out-of-focus phototoxicity with high recording speeds. Here we describe several applications of MMM to live cell imaging using the neuroendocrine cell line PC12 and bovine chromaffin cells. Stainings were performed with the acidophilic dye acridine orange and the lipophilic dyes FM1 - 43 and Fast DiA as well as by transfection of the cells with GFP. In both bovine chromaffin and PC12 cells structural elements of nuclear chromatin and the 3-D distribution of acidic organelles inside the cells were visualized. In PC12 cells differentiated by nerve growth factor examples of neurites were monitored. Stainings of membranes were used to reconstruct the morphology of cells and neurites in three dimensions by volume-rendering and by isosurface plots. 3-D reconstructions were composed from stacks of about 50 images each with a diameter of $30-100 \mu \mathrm{m}$ that were acquired within a few seconds. We conclude that MMM proves to be a technically simple and very effective method for fast 3-D live cell imaging at high resolution.

\section{Introduction}

Microscopy of live cells requires technologies which permit normal functioning of cells under well defined and controlled conditions. Furthermore, the signals need to be specific and long-lasting while simultaneously allowing for high temporal and spatial resolution. During the last decade, two-photon

\footnotetext{
1) M. Straub, High Resolution Optical Microscopy Group, MaxPlanck-Institut für Biophysikalische Chemie, Am Fassberg 11, D37077 Göttingen/Germany; e-mail: mstraub@gwdg.de, Fax: + 49551 2011085.
}

excitation fluorescence microscopy has emerged as a novel, high-resolution microscopic technique that possesses many advantages over confocal microscopy for 3-D imaging of live cells (Denk et al., 1990). Simultaneous absorption of two photons confines fluorescence excitation to the focal region ensuring optical sectioning. Out-of-focus photobleaching and photodamage are strongly reduced (Denk et al., 1995). Therefore, multiphoton excitation microscopy promises to maintain normal functioning of live cells for a much longer time than its one-photon counterpart (Squirrel et al., 1999). Moreover, twophoton and three-photon excitation (Hell et al., 1996; Wokosin et al., 1996) allows the use of UV-excitable dyes without UVoptics. In addition, with tunable infrared lasers (for example the Ti:sapphire laser with a range from 690-1050 nm) the excitation wavelengths of dyes are easier to match than with the discrete wavelengths available in one-photon laser scanning confocal microscopy. The power of two-photon microscopy has recently been documented in applications such as $\mathrm{Ca}^{2+}$-imaging in brain slices (Yuste and Denk, 1995) and in intact cortex (Svoboda et al., 1997), the study of embryonic development (Potter, 1996) and others (an overview is given in (Piston, 1999)).

For many investigations of live cell functions image acquisition at video rate $(25 \mathrm{~Hz})$ or even faster is required, or at least desirable. Video-rate two-photon imaging has been successfully accomplished through increasing the scan speed of a galvanometer scanner (Fan et al., 1999; Tan et al., 1999; Kim et al., 1999). As the available photon flux from the sample is limited, however, increasing the scan speed requires very bright samples, or else confinement of scanning to a small region of the field. Pixel integration times greater than $5 \mu$ s are often necessary in order to achieve an acceptable signal in standard multiphoton excitation microscopy. Therefore, recording a $512 \times 512$ pixel image of a sample with $50-200 \mu \mathrm{m}$ in diameter usually requires several seconds. If stacks of 10-100 sections through a whole cell need to be taken, image acquisition is too slow for the study of many dynamic processes in three dimensions. Another option would be multiphoton line scanning (Brakenhoff et al., 1996), but this technique inevitably trades off the axial resolution against speed. 
The recording time in multiphoton microscopy can be considerably shortened if an array of high numerical aperture foci (typically $\sim 30$ ) simultaneously scans the sample while parallel detection is achieved by a CCD camera. Such a multifocal multiphoton microscopy (MMM) system was first demonstrated with a hexagonal pattern of microlenses mounted on the periphery of a rotating disk (Bewersdorf et al., 1998; Straub and Hell, 1998a). Another approach makes use of a fixed arrangement of square-shaped microlenses generating an array of foci which are scanned over the sample by a galvanometer mirror (Buist et al., 1998). Imaging of larger areas with lower resolution has also been shown with a regenerative amplifier in combination with one to two orders of magnitude higher number of foci (Fujita et al., 1999). Moreover, by confocalizing MMM a further increase in axial resolution as well as strong suppression of scattered fluorescence light may be achieved (Fujita et al., 2000).

Recently, we have demonstrated that MMM is a generally useful tool for 3-D imaging of biological samples including live cells (Straub and Hell, 1998a). MMM also facilitates efficient 3-D lifetime microscopy as exemplified on specimens containing fluorescent beads and tissue culture chinese hamster ovary cells transfected with green fluorescent protein (Straub and Hell, 1998b, 1999). Examples of fast dynamic processes have been given by time-sequences of sections through live boar sperms (Bewersdorf et al., 1998) and by imaging $\mathrm{Ca}^{2+}$ dynamics (Fujita et al., 2000).

In this article we demonstrate the potential of MMM in imaging the morphology of live cells in three dimensions at high resolution with recording speeds up to video rate. Special emphasis is put on their detailed three-dimensional reconstruction. As examples, we use primary cultured chromaffin cells and PC12 cells. These neuroendocrine cells contain acidic storage organelles, the secretory or chromaffin granules, that undergo regulated exocytosis upon stimulation (Henkel and Almers, 1996). Furthermore, PC12 cells respond to stimulation by NGF with further differentiation including outgrowth of neurites associated with a change in cell morphology (Tischler and Green, 1976; Levi et al., 1988). For imaging we used different types of fluorescent dyes suitable for live cell applications. Acridine orange accumulates in acidic organelles and binds to acidic structures such as DNA. Both accumulation and binding are associated with characteristic changes in fluorescence that can be exploited in fluorescence microscopy (Millot et al., 1997). Furthermore, we used the lipophilic dyes Fast DiA and FM1 - 43 that selectively accumulate in bilayer membranes but differ in their partition coefficient. In some cases the cytosol was stained homogeneously by transfecting the cells with GFP. In all experiments the cells were studied in three dimensions resolving laterally $\sim 300 \mathrm{~nm}$ and axially $\sim 1.4 \mu \mathrm{m}$ (Bewersdorf et al., 1998). The images were recorded using exposure times as short as $40 \mathrm{~ms}$ per slice. The results are displayed as sequences of axial sections, as volume-rendered 3$D$ reconstructions, and as isosurface plots calculated from the image stacks.

\section{Materials and methods}

\section{Microscope setup}

The experiments were carried out with a mode-locked Titanium:Sapphire (Ti:Sa) laser (Mira 900-F, Coherent, Palo Alto, CA) providing 140 -fs pulses at a repetition rate of $76 \mathrm{MHz}$ with $\sim 2 \mathrm{~W}$ average power.
The Ti: Sa was pumped by $14 \mathrm{~W}$ continuous wave power from an argon ion laser (INNOVA 410, Coherent).

Figure 1 shows a photograph of the MMM system (a) as well as a sketch of the experimental setup (c). The Ti:Sa beam was expanded by a zoom telescope, and its edges were cut off by a stop in order to ensure sufficiently homogeneous illumination of the field of view. It then impinged onto a rotating microlens disk the optical properties of which have been described in detail elsewhere (Bewersdorf et al., 1998; Straub and Hell, 1998a). The microlens disk splits the beam into 20-50 single beamlets which were focussed in a plane located $4.5 \mathrm{~mm}$ behind it. The array of foci was then imaged further onto the sample via intermediate optics which allowed the distance between the foci on the sample to be varied. The focal plane was completely scanned within $2.7 \mathrm{~ms}$ with the density required by the Nyquist criterion, so that a full and uniform coverage of the field of view was ensured. Fluorescence light passed a dichroic mirror and was detected by a CCD camera or could be directly viewed by eye. The camera was an Imager $3 \mathrm{~L}$ (LaVision, Göttingen) featuring a Sony Interline VGA chip cooled to $-15^{\circ} \mathrm{C}$, a dynamic range of $12 \mathrm{bit}$, read out rate $12.5 \mathrm{MHz}$, and a readout noise of $\sim 13 \mathrm{e}^{-}$. The quantum efficiency of the chip was 35$40 \%$ at $400-550 \mathrm{~nm}$ and $\sim 20 \%$ at $650 \mathrm{~nm}$, its pixel size was $9.9 \mu \mathrm{m}$. Images were recorded with water immersion objectives (Olympus Uplan APO, $60 \times$, or Leica PL APO, $63 \times$, both NA 1.2), for smaller objects in combination with a $1.6 \times$ tube lens, resulting in magnifications between 60 and 96 . In order to block reflections of laser light suitable filter combinations were placed in front of the camera. The filter stack contained custom-made interference filters, transmitting below $750 \mathrm{~nm}$ while attenuating by five orders of magnitude above. Additional bandpass and edge filters were used as emission filters. The most important parameters concerning sample preparation and imaging such as the staining conditions, the excitation wavelength, number of foci and average power in a focus, magnification, exposure time and emission filters used are indicated in Table I for each recording.

\section{Cell culture and transfection}

PC12 cells were maintained in 25-cm culture dishes containing RPMI growth medium (with $28 \mathrm{mM}$ glucose, $5 \%$ FCS, $10 \% \mathrm{HS}, 4 \mathrm{mM} \mathrm{L}-$ glutamine, $60 \mathrm{U} / \mathrm{ml}$ penicillin and streptomycin; $\mathrm{pH} 7.4$ ) at $37^{\circ} \mathrm{C}$ in $10 \%$ $\mathrm{CO}_{2} .48 \mathrm{~h}$ prior to experiments the cells were split and grown on poly-Llysine-coated glass coverslips at an average density of $\sim 10^{5} / \mathrm{ml}$. They were differentiated with rat recombinant nerve growth factor (Sigma, Deisenhofen, Germany) added to the growth medium at a concentration of $50 \mathrm{ng} / \mathrm{ml}$ two to seven days before the experiments. Bovine chromaffin cells were prepared as described elsewhere (Smith et al., 1998). For transfections, cells were detached from their substrates and resuspended in cytomix $\left(120 \mathrm{mM} \mathrm{KCl}, 10 \mathrm{mM} \mathrm{KK_{2 }} \mathrm{PO}_{4}, 0.15 \mathrm{mM}\right.$ $\mathrm{CaCl}_{2}, 2 \mathrm{mM}$ EGTA, $5 \mathrm{mM} \mathrm{MgCl}, 2 \mathrm{mM}$ ATP, $5 \mathrm{mM}$ gluthatione, and $25 \mathrm{mM}$ k-HEPES ( $\mathrm{pH}$ 7.6)). Cytomix ( $350 \mu \mathrm{l})$ containing $2 \times 10^{6}$ cells was placed into an electroporation cuvette, then $50 \mu \mathrm{g}$ pEGFP-C1 (Clontech, Cat\#6084-1) was added. A single 1150-V shock was applied through $50 \mu \mathrm{F}$ and $24 \Omega$. Cells were placed onto glass coverslips and used for experiments $48 \mathrm{~h}$ later.

\section{Staining}

Staining with acridine orange, FM1-43 and Fast DiA (all Molecular Probes, Eugene, OR; for the structure formula see Table II) was performed in a temperature-controlled superfusion microscope chamber shown in Fig. 1b. Immediately prior to the experiments the cells were washed in Krebs-Ringer solution ( $142 \mathrm{mM} \mathrm{NaCl}, 25 \mathrm{mM}$ HEPES, pH 7.4, $5.6 \mathrm{mM}$ glucose, $4.8 \mathrm{mM} \mathrm{KCl}, 1.3 \mathrm{mM} \mathrm{CaCl}_{2}, 1.2 \mathrm{mM} \mathrm{KH}_{2} \mathrm{PO}_{4}$, $1.2 \mathrm{mM} \mathrm{MgSO}_{4}$ ). They were stained at $37^{\circ} \mathrm{C}$ by perfusion of the chamber with the dyes diluted either in Krebs-Ringer buffer or high potassium stimulation buffer (as Krebs-Ringer, but $104 \mathrm{mM} \mathrm{KCl}$, $25 \mathrm{mM} \mathrm{NaCl}$ ) or by adding the dye to the culture media. In some experiments (see Fig. 6) the plasma membrane was stained at $4{ }^{\circ} \mathrm{C}$ to keep internalization of dye low. The incubation periods are as indicated. 

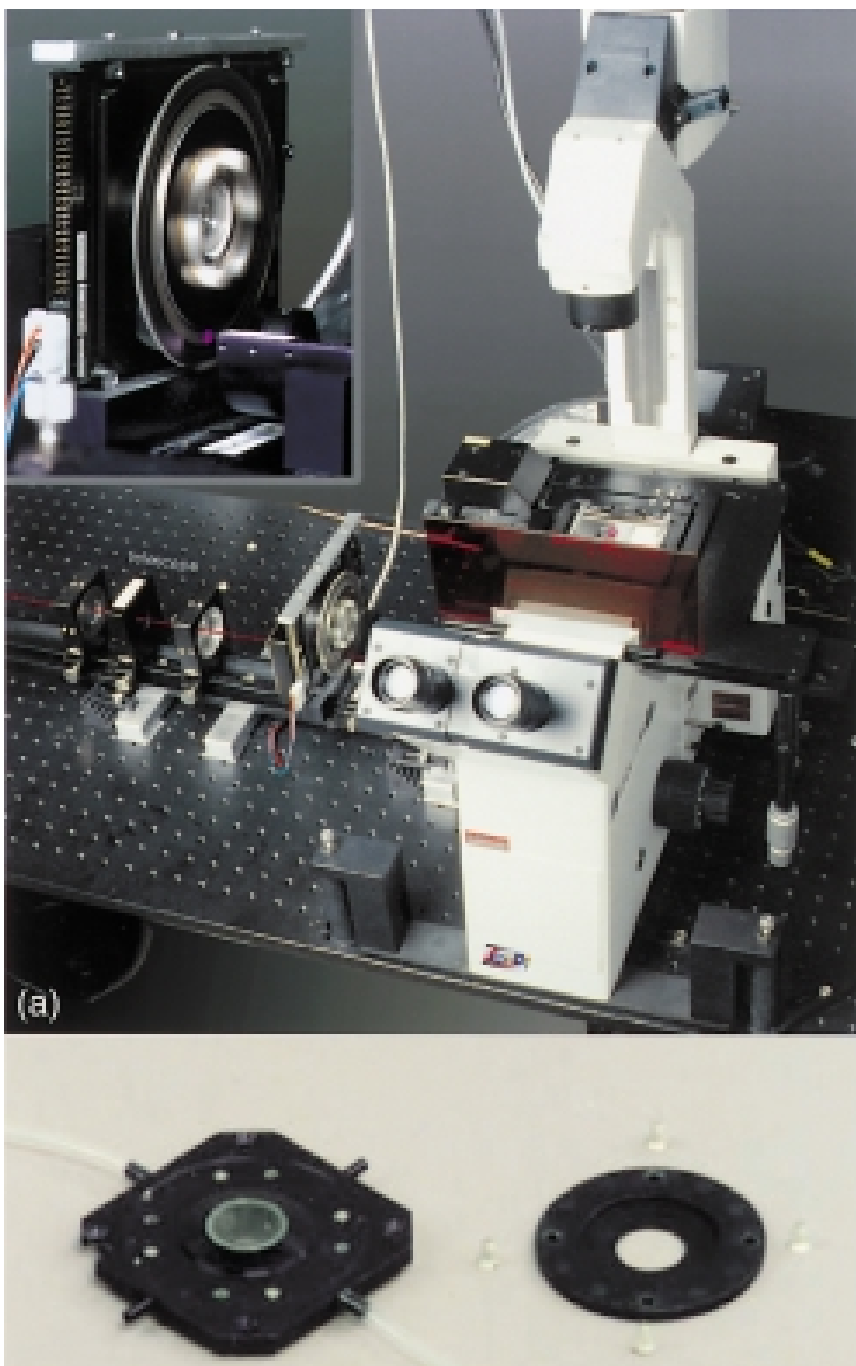

(b)

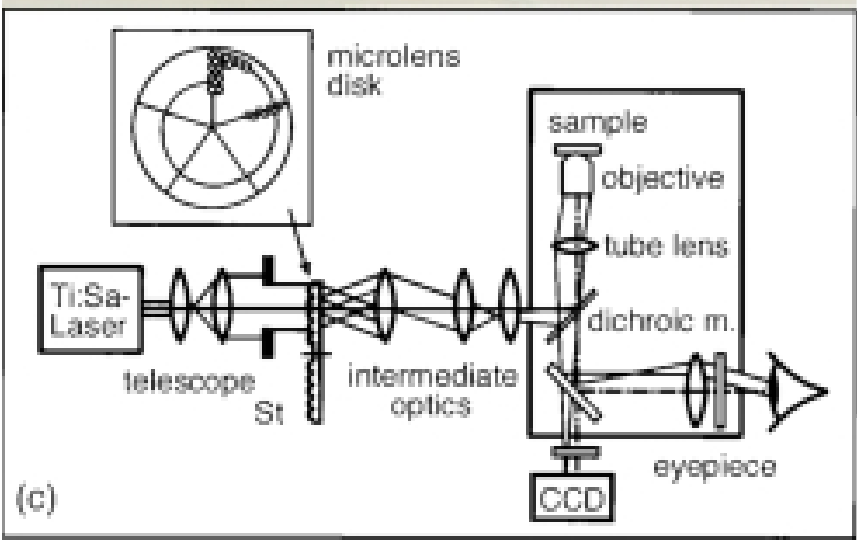

\section{Results}

\section{Bovine chromaffin cells}

Images of live bovine chromaffin cells stained for 3 min with $10 \mu \mathrm{M}$ acridine orange are shown in Fig. 2. Acridine orange exhibits characteristic spectral changes when bound to acidic structures or present in acidic compartments, a property which
4 Fig. 1. Photographs showing (a) the multifocal multiphoton microscope and (b) the temperature controlled superfusion microscope chamber. A sketch of the optical layout of the microscope is given in (c). The Ti:Sapphire laser beam is expanded by a zoom telescope and cut by a stop before being split by the microlens disk into about 30 beamlets which are imaged onto the sample as an array of high numerical aperture foci. Fluorescence is detected either by a CCD camera located below the microscope or directly through the eyepiece. The spinning microlens disk is shown as an inset together with part of the adjoining optics. The microlenses (diameter $460 \mu \mathrm{m}$ ) are arranged in a hexagonal pattern on its periphery, intermediate space between them is obstructed by a thin metal foil. The coverslips were mounted onto the O-ring gasket of the chamber which was closed by fixing the cap with screws (b). On the microscope it was then inserted into an isolating carrier and covered by a digitally controlled heater plate (not shown).

makes it a useful reagent in histology for staining nucleic acids (Clerc and Barenholz, 1998; Darzynkiewicz, 1990; Petit et al., 1993). In addition, it is used for staining acidic organelles, e.g. lysosomes or neuroendocrine secretory granules (Steyer et al., 1997). Bound to DNA, acridine orange has its emission maximum in the green $(\lambda \sim 525 \mathrm{~nm})$, whereas when concentrated in acidic organelles it shifts into the red $(\lambda \sim 650 \mathrm{~nm}$; Millot et al., 1997). In order to monitor the distribution of acidic compartments in the whole cell with sufficiently high spatial and temporal resolution, stacks of axial sections were recorded and subjected to 3-D reconstructions.

The staining pattern shown in Fig. 2 is typical for a bovine chromaffin cell. The six images in steps of $2.5 \mu \mathrm{m}$ represent an assortment of a stack consisting of 51 images through the whole cell. They are presented as two-channel recordings. The nuclei were recorded with an interference bandpass filter in the green (D520/60, Chroma, Brattleboro, VT), whereas for the vesicular structures emitting in the red an OG590 edge filter (Schott) was applied. The exposure time per slice was $500 \mathrm{~ms}$ for the red channel. The green component was recorded within $2.0 \mathrm{~s}$ improving the signal-to-noise ratio in order to catch details of the nucleus more clearly. Both images were combined using the two-dimensional colour table shown in Fig. 2b. The vertical axis represents the colour table for the red channel, the horizontal axis a second one for the green channel. Both colour tables were combined by summing their $\mathrm{r}-$, g-, and b-values, respectively. The resulting rgb-colour is indicated inside the 2-D colour table. The distribution of acidic compartments (red) all around the nucleus is recognized with a high lateral and axial resolution. Acidic compartments appear to be wide-spread throughout the cytoplasm with regions of relatively high concentration. However, single organelles are hard to resolve due to the high density of acidic compartments. In this respect, it should be noted that bovine chromaffin cells contain several 10000 acidic secretory granules with an average size of $365 \mathrm{~nm}$ (Plattner et al., 1997). As seen from the $2 \mathrm{D}$-colour table both the nucleus and the acidic compartments contribute to the fluorescence signal in the yellow coloured perinuclear zone. Regions in the nucleus with a very high signal in the green channel (blue structures) may be zones of highly concentrated euchromatic DNA.

In order to visualize the 3-D data set as a whole the method of volume rendering was applied. For this purpose we used a special software package (AMIRA 3-D Visualization and Modelling System, Konrad-Zuse-Zentrum für Informations- 
Tab. I. Parameters used for preparation and observation in each experiment.

\begin{tabular}{|c|c|c|c|c|c|c|c|c|c|c|}
\hline \multirow[t]{2}{*}{ Figure } & \multirow[t]{2}{*}{ Sample } & \multicolumn{3}{|l|}{ Staining } & \multirow{2}{*}{$\begin{array}{l}\text { Wave- } \\
\text { length } \\
(\mathrm{nm})\end{array}$} & \multirow{2}{*}{$\mathrm{N}_{\text {foci }}$} & \multirow{2}{*}{$\begin{array}{l}\text { Power/ } \\
\text { focus } \\
\text { (mW) }\end{array}$} & \multirow{2}{*}{$\begin{array}{l}\text { Magni- } \\
\text { fication }\end{array}$} & \multirow{2}{*}{$\begin{array}{l}\text { Exposure time } \\
\text { per slice }(\mathrm{s})\end{array}$} & \multirow{2}{*}{$\begin{array}{l}\text { Emission } \\
\text { filters }\end{array}$} \\
\hline & & Dyes & $\begin{array}{l}\text { Conc. } \\
(\mu \mathrm{M})\end{array}$ & $\begin{array}{l}\text { Incub. } \\
\text { (min) }\end{array}$ & & & & & & \\
\hline 2,3 & Bovine chromaffin cell & Acridine orange & 10 & 3 & 850 & 30 & 4.0 & $96 \times$ & 0.5 (red), 2.0 (green) & D520/60, OG590 \\
\hline & NFG-differentiated PC12 cell & Acridine orange & 30 & 5 & 840 & 30 & 3.5 & $60 \times$ & & D520/60, OG590 \\
\hline $5(a)$ & Growth cone & Fast DiA & 10 & 15 & 870 & 30 & 3.0 & $96 \times$ & 4.0 & - \\
\hline $5(b)$ & Varicosity & $\mathrm{FM} 1-43$ & 30 & 2 (stim.) & 870 & 30 & 3.0 & $96 \times$ & & \\
\hline 6 & NFG-differentiated PC12 cells & Fast DiA, EGFP & 30 & 5 & 880 & 50 & 3.0 & $63 \times$ & $0.5(a-c), 0.04(d)$ & D580/60, D510/40 \\
\hline
\end{tabular}

Tab. II. Structure formulas of the fluorescent dyes used with absorption and emission maxima.

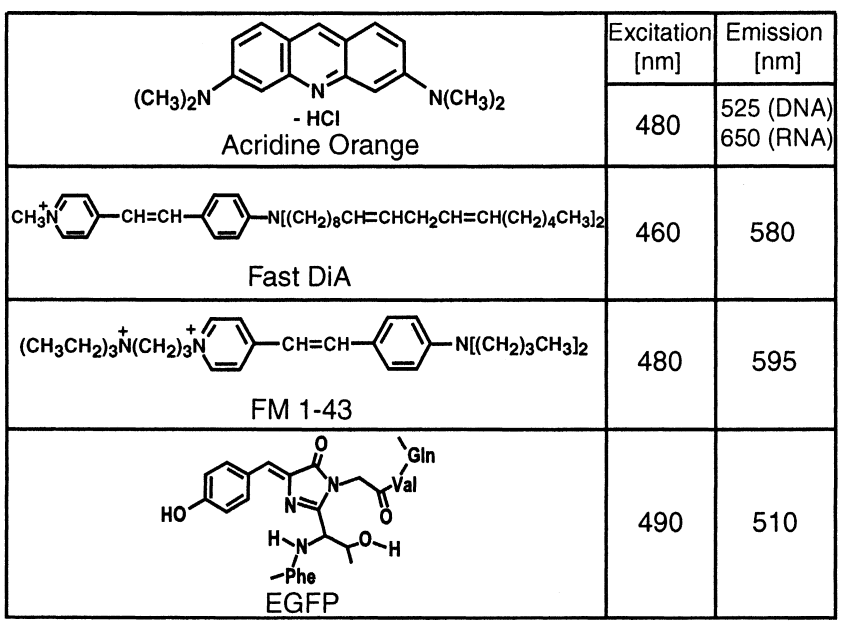

The cationic dye acridine orange interacts with DNA and RNA by intercalation or electrostatic interaction and concentrates in acidic organelles in a pH-dependent manner. The lipophilic aminostyryl dye FM1-43 and the polyunsaturated styryl dye Fast DiA reversibly stain membranes. The cytosol was stained homogeneously by the enhanced green fluorescent protein (EGFP, Clontech).

technik, Berlin) which reconstructs the 3-D structure and generates various 2-D projections. From the original data the program calculates a stack of up to $\sim 250$ slices with adjustable transparency, which can be oriented arbitrarily, to make up a scattering object illuminated by a point source. A background of about $5 \%$ is subtracted. In Fig. 3a two such reconstructions of 51 images acquired with different emission filters are combined into a two-colour 3-D image. Fig. $3 \mathrm{~b}$ depicts projections of the cell from three different viewing angles. This way of presentation has the advantage that the precise geometrical arrangement of the cell components is now visualized much better. Note especially, that the arrangement of the acidic compartments as well as the different patterns in the nucleus can be recognized in more detail. Fig. $3 b$ shows the cell from panel (a) as rotated images, first by $45^{\circ}$ around the z-axis and then $20^{\circ}$ around the $y$-axis (left), and also by $35^{\circ} / 25^{\circ}$ (middle) and $180^{\circ} / 30^{\circ}$ (right).

After volume rendering, zones of highly accumulated dye can clearly be distinguished in the nucleus from zones with much weaker staining (compare Figs. $2 \mathrm{a}$ and $3 \mathrm{~b}$ ). These correlate with the bluish/white and greenish patterns in Fig. 2a. As previously reported (Delic et al., 1991), the acridine orange staining is characteristic for the nonpermissive state of the chromatin, a feature of the nucleus in living cells. The small fluorescent object outside the bovine chromaffin cell is probably a lymphocyte, a frequent contamination of the cell preparation.

It is worthwhile to note that by comparing the projection of the 3-D reconstruction in panel (a) with those in (b), the difference in lateral and axial resolution in MMM becomes obvious. The lateral resolution equals that of a conventional microscope of approximately $300 \mathrm{~nm}$ for our water immersion objective (NA 1.2) at $\lambda \sim 550 \mathrm{~nm}$. Note that this is slightly better than that of a scanning two-photon system using $850 \mathrm{~nm}$ and roughly equals that practically obtained in a confocal laser scanning microscope. In contrast, the axial resolution is largely determined by the axial widths of the two-photon point spread function of a single water immersion lens; it amounts to about $1.4 \mu \mathrm{m}$ (Bewersdorf et al., 1998). For this reason a point-like object in a 3-D reconstruction resembles a rotationally symmetric ellipsoid with a ratio between its semiaxes around five. In Fig. 3a the bovine chromaffin cell is projected onto an xy-plane along the $\mathrm{z}$-axis. Thus, all structures are displayed with the high lateral resolution. In particular, many details within the clusters of acidic compartments can be resolved. Naturally, when rotating the 3-D reconstruction and inspecting it from the side, the resolution is five times poorer. However, it should be pointed out that by confocalizing MMM (Fujita et al., 2000) or combining it with 4Pi-microscopy (Hell et al., 1997) a significant increase in axial resolution can be achieved.

\section{NGF-differentiated PC1 2 cells}

In the following experiments, we used different staining procedures to image live NGF-differentiated PC12 cells in order to illustrate the versatility of MMM for addressing dynamic aspects of cell biology. Fig. 4 shows the result of labelling with acridine orange ( $30 \mu \mathrm{M}$ dye, 5 min of labelling). A cell body is depicted with some processes extending out of the field of view. Red and green components of fluorescence are presented separately by (a) volume rendering and (b) as sequences of xy-slices. The cell is increased in size when compared to an undifferentiated PC12 cell (not shown) and a relatively spotty pattern of acidic compartments throughout the cell body is observed. Spots seemingly accumulate in distinct perinuclear regions. The exposure time per xy-slice was $200 \mathrm{~ms}$.

In Fig. 5, we used lipophilic dyes in order to label membranes of PC12 cell neurites. NGF is known to induce the formation of very large, growth cone-like neurites that may serve as a model for growth cones in neurons. First, staining was performed with Fast DiA (dye concentration $10 \mu \mathrm{M}$, labelling time $15 \mathrm{~min}$ ). This dye partitions into membranes and remains in the membrane even when excess dye is removed by washing. Thus, DiA is ideally suitable to visualize all accessible 

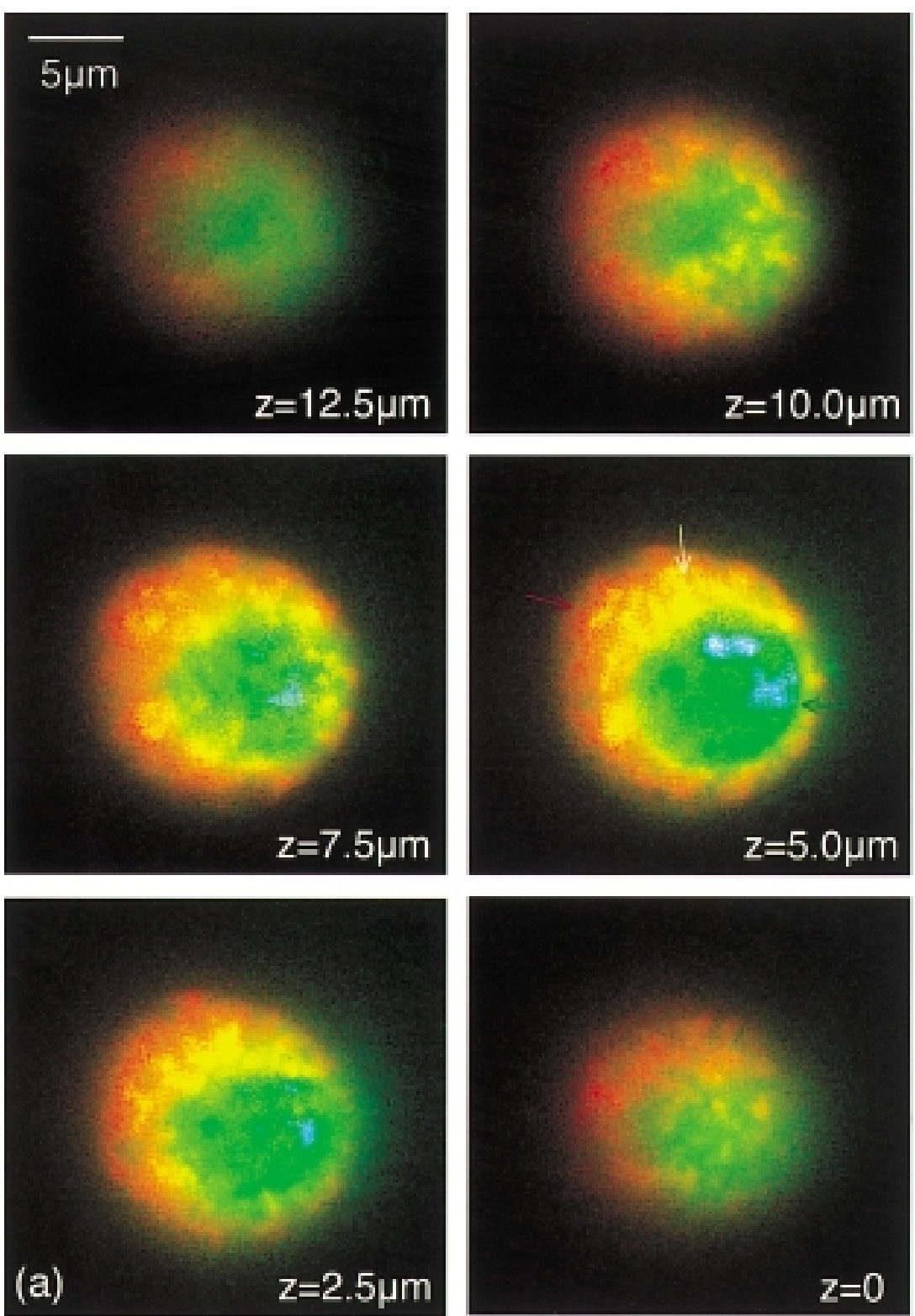

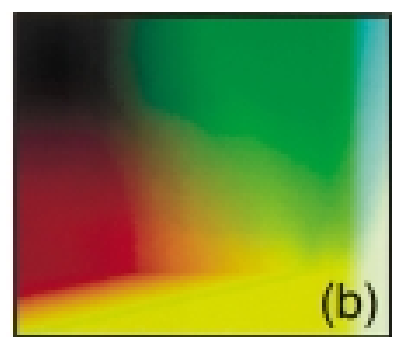

(b)
Fig. 2. Sequence of sections through a bovine chromaffin cell in steps of $2.5 \mu \mathrm{m}$ (a). The cell was stained with $10 \mu \mathrm{M}$ acridine orange for $3 \mathrm{~min}$. Green fluorescence $(\lambda \sim 525 \mathrm{~nm})$ is emitted by DNAbound dye in the nucleus, whereas red fluorescence $(\lambda \sim 650 \mathrm{~nm})$ is generated inside acidic organelles. The images are presented as two-colour recordings using a two dimensional colour table (b). The vertical axis represents the red channel, the horizontal axis the green channel. For the red fluorescence the exposure time was $500 \mathrm{~ms}$, for the green fluorescence $2.0 \mathrm{~s}$. Acidic compartments inside the cell, the perinuclear zone and the nucleus are marked by red, yellow and green arrows, respectively. membranes, i.e. the plasma membrane and internal membranes that are connected with the plasma membrane by endocytotic membrane flow during the incubation time. The precise outline of the neurite plasma membrane was revealed (exposure time $4.0 \mathrm{~s}$ ) (Fig. 5a). Numerous, needle-like sproutings of filopodia, up to $15 \mu \mathrm{m}$ in length, were observed demonstrating the high axial resolution of MMM. Their width as seen in the images is determined by the effective focal spot size. Rotating such structures around various axes on our graphics work station showed that some of the needle-like structures projected at different angles out of the plane of the coverslip. This is also recognized in the 3-D reconstruction. Sproutings of filopodia may function at particular phases of growth cone movement, for example as the neurite approaches its target cell, or in response to electrical stimulation (Manivannan and Terakawa, 1994). In some sections, brightly fluorescent spots are seen close to the outer surface of the cell, probably due to endocytotic internalization of the dye during the incubation time. The long process at the upper right edge is the connecting branch to the cell body, which is not in the field of view, and appears somewhat fainter near the border of the image due the Gaussian profile of the laser beam.

Second, we performed staining of a neurite with the lipophilic dye FM1 - 43. In contrast to DiA which partitions almost exclusively in the lipidic phase, FM1-43 exists in a dynamic equilibrium between the aqueous and the membrane phase and is therefore washed out in seconds. Furthermore, fluorescence is low in water and greatly increases upon binding to a membrane. The dye molecule cannot cross the membrane, but is taken up by endocytosis during incubation. Thus, the dye is ideally suitable to visualize endocytosed organelles without interference by labelling of the plasma membrane. Neurites of PC12 cells were labelled with FM1 - 43 for 2 min. Labelling was performed in depolarizing, high-potassium buffer, i.e. conditions under which exo-endocytotic membrane recycling is stimulated. FM1 - 43 was then washed out for 10 minutes in normal Krebs-Ringer buffer. This leads to a staining of recycling membrane compartments as is particularly well 


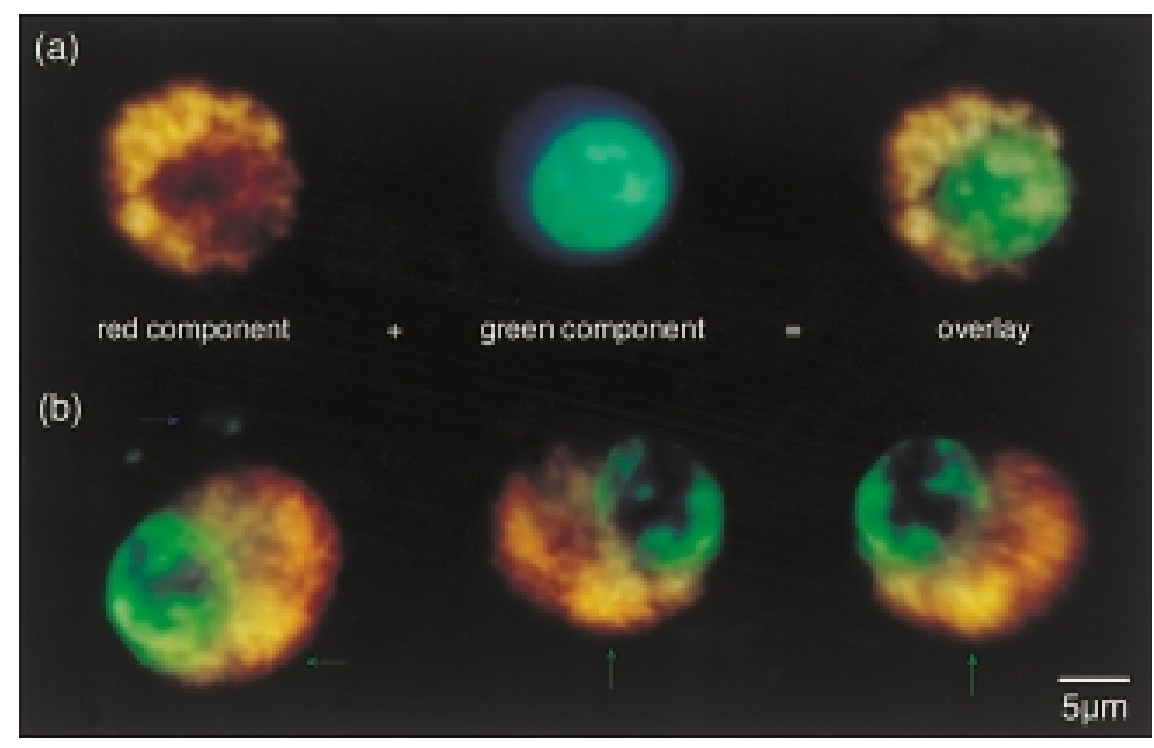

Fig. 3. Same bovine chromaffin cell as depicted in Fig. 2. Panel (a) illustrates how volume-rendered reconstructions of the red and the green components of fluorescence light (left) are combined to a two-colour reconstruction (right) which is shown from three different perspectives $(\mathbf{b})$. Note the high concentration of acidic compartments in perinuclear regions (arrow) and the stained lymphocyte in the background.
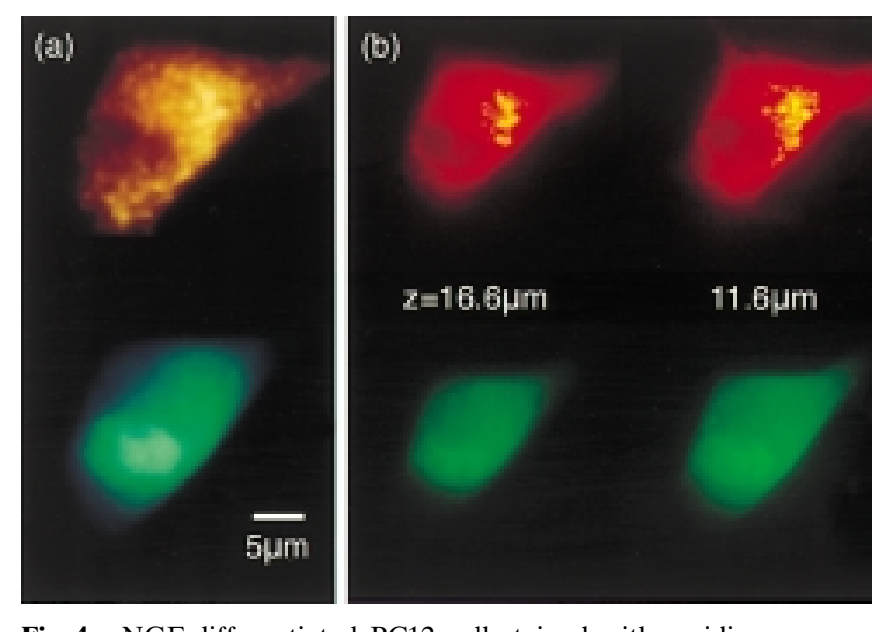

Fig. 4. NGF-differentiated $\mathrm{PC} 12$ cell stained with acridine orange. Fluorescence of the nucleus (green) and of acidic organelles (red) are distinguished. The upper part of the figure presents a volume-rendered reconstruction of the red component emission pattern (a) together with

demonstrated in this series of axial sections (Fig. 5b). Many small punctate structures can be observed intermingled with larger, clustered objects.

In the final series of experiments, we demonstrate that MMM can be used to obtain a detailed 3-D contour surface map of cells (Fig. 6). Isosurfaces allow analysis of arbitrary scalar fields sampled on a discrete grid and provide a fast method for reconstructing polygonal surface models. Fractional weights generated automatically during segmentation result in smooth boundary interfaces. To illustrate this feature, PC12 cells differentiated by NGF were transfected with green fluorescent protein (GFP) to achieve a homogeneous fluorescence background by the cytosol. In addition, they were labelled with $\mathrm{DiA}(30 \mu \mathrm{M}$ for $5 \mathrm{~min})$ at a temperature of only $4{ }^{\circ} \mathrm{C}$ in order to prevent the dye from penetrating into the interior of the cells. Membrane and volume stainings were recorded separately using D580/60 and D510/40 interference bandpass filters (Chroma). $32 \mathrm{MMM}$ images were acquired in steps of $830 \mathrm{~nm}$ with exposure times of $500 \mathrm{~ms}(\mathrm{a}-\mathrm{c})$ and $40 \mathrm{~ms}$ per slice (d). Four representative slices are depicted in panel (b) showing the DiA component (upper series) as well as the GFP component (lower series). It is clearly recognized that the $\mathrm{DiA}$ is concentrated at the plasma membrane revealing the precise course of the neurites, whereas the interior of the cell is stained mainly by GFP with some vacuoles visible in the two uppermost sections. The GFP distribution was reconstructed by volume rendering, while based on the DiA staining an isosurface plot was generated. For both reconstructions the data sets used were compensated for the Gaussian intensity distribution of the laser beam, which led to a decay of fluorescence to about $40 \%$ near the edge of the images. Panel (a) shows a combined 3-D representation of both reconstructions both in the axial view and tilted by various angles around a vertical and a horizontal axis. The morphology of the membranes is resolved in great detail. Several filopodia and neurite outgrowths are visible. By varying the surface data threshold, information about the distribution of fluorescent staining could be obtained. 


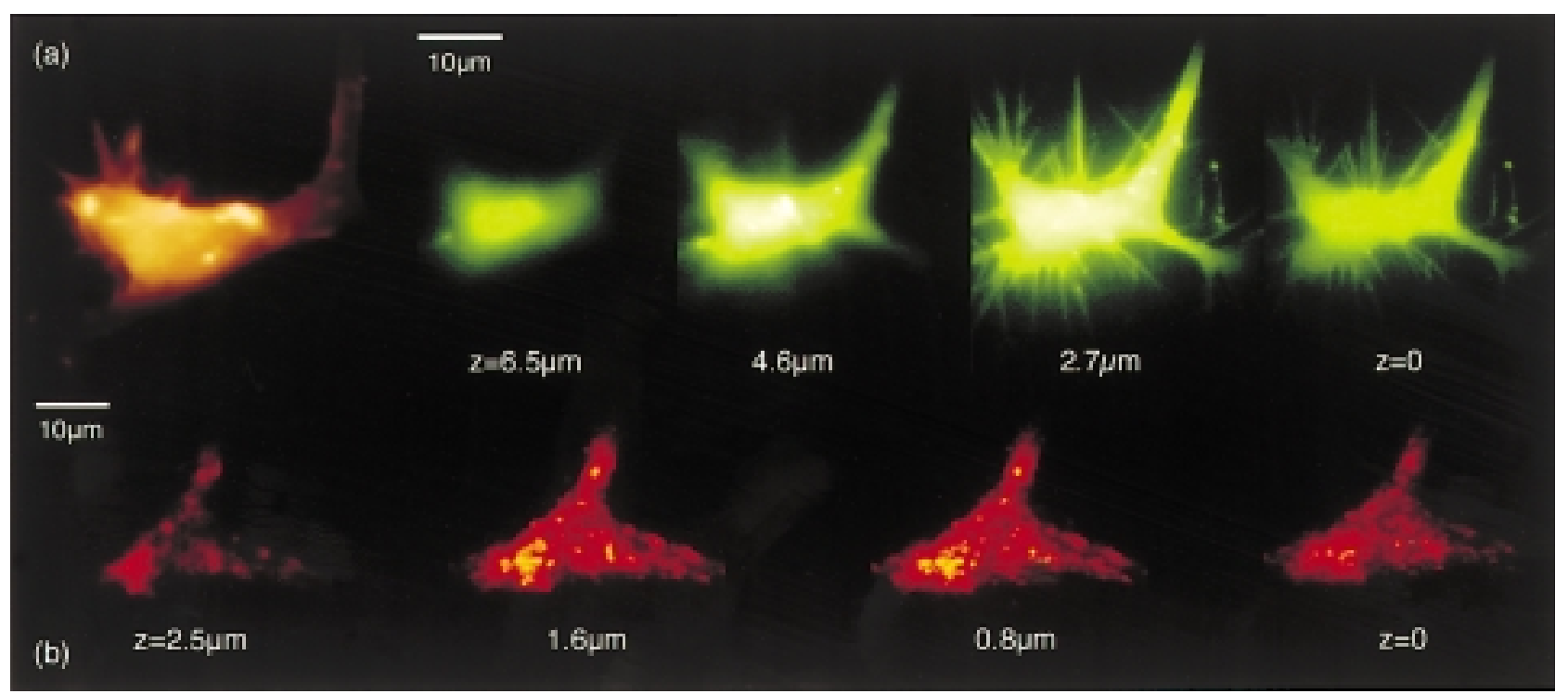

Fig. 5. On exposure to nerve growth factor (NGF) PC12 cells differentiate into neuron-like cells developing neuritic outgrowths. (a) End of an advancing neurite of an NGF-differentiated PC12 cell stained with $10 \mu \mathrm{M}$ Fast DiA for $15 \mathrm{~min}$, shown as volume-rendered reconstruction (left) and as four representative slices (right). The 3-D reconstruction was generated from 20 slices recorded in steps of $950 \mathrm{~nm}$ at an exposure time of $4.0 \mathrm{~s}$ each. The tiny processes reveal the high axial resolution of MMM of up to $1.4 \mu \mathrm{m}$. (b) Series of axial sections through the branched varicosity of an NGF-differentiated PC12 cell. Staining was performed by a 2-min incubation with $30 \mu \mathrm{M}$ FM1 - 43 in stimulation buffer followed by 10 minutes wash. Bright punctate fluorescence can be seen throughout the structure indicative of high membrane turnover $\left(\mathrm{t}_{\exp }=4.0 \mathrm{~s}\right)$.
Panel (c) depicts an isosurface plot of only the DiA component of an NGF-differentiated $\mathrm{PC} 12$ cell with an interesting topology. Several neurite outgrowths, up to $5 \mu \mathrm{m}$ in diameter, point perpendicularly out of the cell. Most of them are branched at their ends.

In contrast, in panel (d) a combined volume- and surface reconstruction of a cell is shown which was recorded at video rate. In this case only the base filter combination was used, DiA- and GFP contributions not being separated. It is seen that while in general the quality of the image is still very high, small and weekly fluorescent details of the membrane are not resolved because an even higher fluorescence photon flux and a more sensitive camera system would have been required. Importantly, such an improvement can be achieved in MMM, as discussed in the following section.

\section{Discussion}

High resolution microscopy of live cells has begun to dramatically change our perspectives of cell function and cell dynamics. The introduction of novel dyes and, in particular, the availability of fluorescent proteins that can be attached to any cellular protein using molecular tools has revolutionized our approach to cell biology. Therefore, techniques that allow the imaging of thin sectional planes with high spatial and temporal resolution while reducing cell exposure times are in high demand. MMM appears to be an efficient solution for meeting these requirements for numerous applications. It combines high resolution with rapid scanning time while minimizing radiation damage of the sample in comparison to conventional fluorescence microscopy procedures. With respect to damaging MMM is similar to single-beam two-photon microscopy, because in MMM the intensity per focus is the same, and the high number of foci is compensated by a corresponding reduction in exposure time.

In this study we have presented basic examples of live cell imaging to illustrate the potential of MMM. For bovine chromaffin cells stained with acridine orange details of the chromatin structure were resolved, and a rapid overview over the arrangement of acidic organelles was obtained in three dimensions with high resolution. The combination of high spatial resolution with a high recording speed should render MMM a useful tool for studying vesicle dynamics, for instance exocytosis and endocytosis where confinement to a plane can presently only be obtained by evanescent wave microscopy (Stout and Axelrodt, 1989; Steyer et al., 1997; Steyer and Almers, 1999). However, in evanescent wave microscopy observations are restricted to a hundred nanometer zone of the cell which is adjacent to the coverslip.

NGF-stimulated PC12 cells were used to exemplify the power of MMM in determining the shape of live cells and cell processes as well as the distribution of endocytic organelles. Here, MMM is of great advantage as a fast imaging technique because of rapid changes in shape and position of parts of the cells with time. For instance, regions with high membranic turnover were easily identified.

There are many demanding applications expected to benefit from MMM. For example, cellular transport of GFP-labelled vesicles or detection of molecular interaction by fluorescence resonance energy transfer between GFP variants can be monitored. Other applications of MMM may include measurements of rapid changes of the ion concentration in living cells as such applications require high temporal resolution. Moreover, it may be combined with the patch clamp technique (Sakmann and Neher, 1995) allowing simultaneously fluorescence live cell microscopy and electrophysiological measurements. 


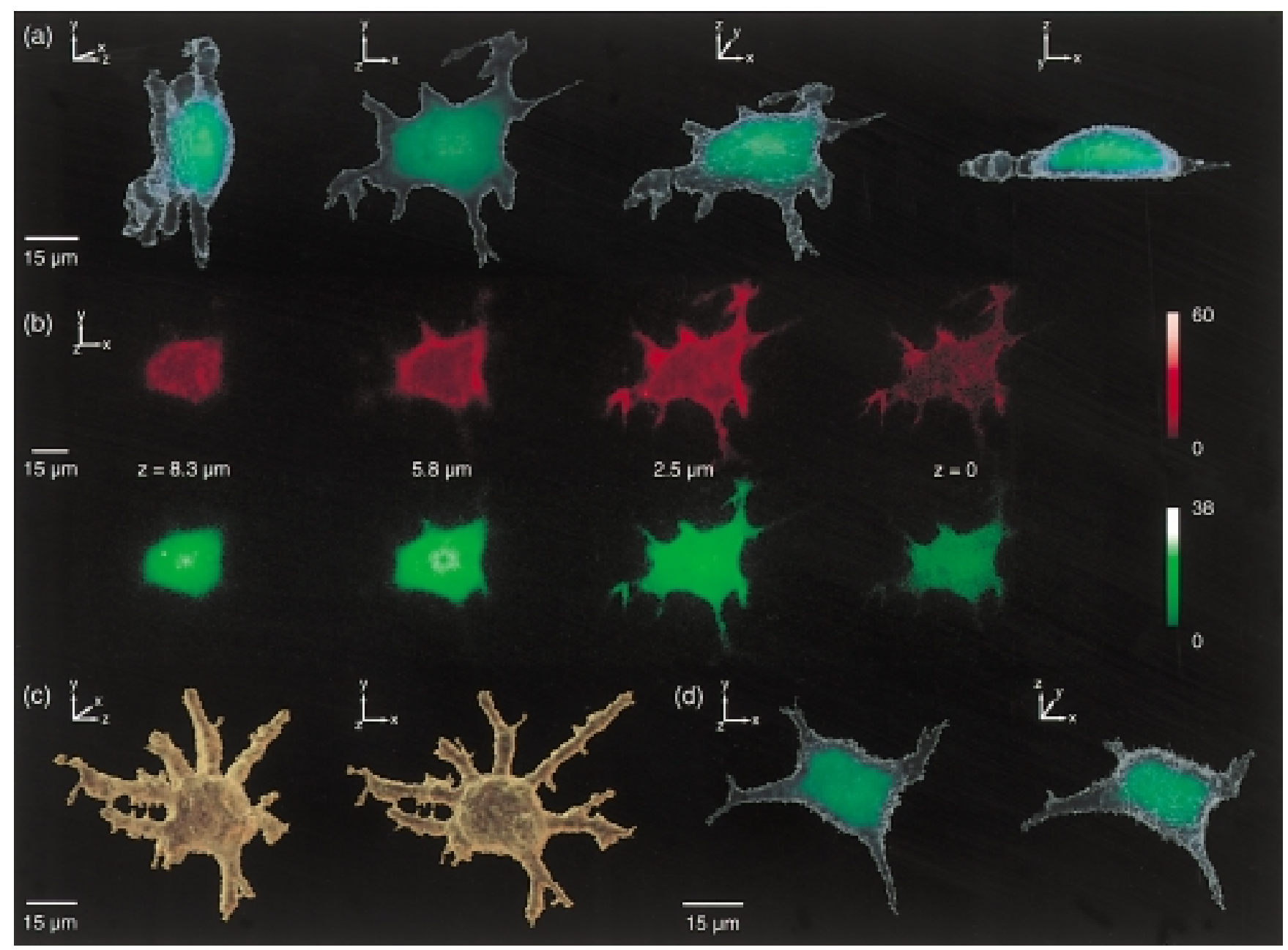

Fig. 6. NGF-differentiated PC12 cells transfected with GFP and their plasma membrane counterstained by Fast DiA. (a) Combined volumerendered reconstruction of the GFP component and isosurface plot of the DiA staining shown at different angles: rotated by $60^{\circ}$ around a vertical axis (first image), axial view (second), rotated by $45^{\circ}$ (third) and $90^{\circ}$ (fourth) around a horizontal axis. The morphology of the cell membrane is resolved in detail. (b) Four representative axial sections for the DiA (upper series) and the GFP staining (lower series). (c) Isosurface reconstruction of the $\mathrm{DiA}$ component recorded from a cell with a particularly complex neurite structure. The 3-D presentations were composed from 32 slices acquired in steps of $830 \mathrm{~nm}$ with $500 \mathrm{~ms}$ exposure time each. (d) Combined volume-rendered/isosurface reconstruction of a cell recorded at video rate without further emission filters. Note the reduced signal-to-noise ratio.
The sensitivity of MMM strongly depends on that of the camera used. Our CCD camera represented a reasonable compromise between read-out speed, cost, and sensitivity. Most recordings in our article have been performed at exposure times of a few hundred milliseconds to a few seconds, in order to resolve also very weekly fluorescent details. However, it should be pointed out that recording times may still be reduced considerably without compromising the resolution of MMM, as required for the study of fast processes. The fluorescence flux can be increased for example by temporal and spatial pulse shaping as well as by the consequent use of infrared antireflection coatings for all parts of the optics, including the microlens disk and the objective. Camera systems with higher quantum efficiency, especially in the red part of the optical spectrum, and read-out speed in the order of $100 \mathrm{~ms}$ are already available. Future development of cameras based on CMOS technology is expected to further strongly shorten exposure times. Intensified cameras also may accelerate image acquisition in specific applications. In contrast, back-illuminated CCDs will be useful if larger integration times can be afforded.

In conclusion, MMM is readily used for imaging live biological specimens with high temporal and spatial resolution. Particularly when combined with sensitive cameras, the rate of light-induced damage is low and a high degree of experimental flexibility is available.

Acknowledgements. We would like to thank the Konrad Zuse Zentrum for Information Technology in Berlin for the Amira 3-D visualization program, Uri Aschery (E. Neher's lab) for providing us with bovine chromaffin cells and Olympus Hamburg for lending us their water immersion objective. We thank T. Jovin for making available to us the technical drawings of the superfusion chamber and T. Lang for useful discussions and critical reading of the manuscript. 


\section{References}

Bewersdorf, J., Pick, R., Hell, S. W. (1998): Multifocal multiphoton microscopy. Opt. Lett. 23, 655-657.

Brakenhoff, G. J., Squier, J., Norris, T., Bliton, A. C., Wade, M. H., Athey, B. (1996): Real-time two-photon confocal microscopy using a femtosecond, amplified Ti:sapphire system. J. Microsc. 181, 253 259.

Buist, A. H., Müller, M., Squier, J., Brakenhoff, G. J. (1998): Real time two-photon absorption microscopy using multipoint excitation. J. Microsc. 192, 217-226.

Clerc, S., Barenholz, Y. (1998): A quantitative model for using acridine orange as a transmembrane $\mathrm{pH}$ gradient probe. Anal. Biochem. 259, $104-111$.

Darzynkiewicz, Z. (1990): Differential staining of DNA and RNA in intact cells and isolated cell nuclei with acridine orange. Methods Cell Biol. 33, 285-298.

Delic, J., Coppey, J., Magdalenat, H., Coppey-Moisan, M. (1991): Impossibility of acridine orange intercalation in nuclear DNA of the living cell. Exp. Cell Res. 194, 147-153.

Denk, W., Piston, D. W., Webb, W. W. (1995): Two-photon molecular excitation in laser scanning microscopy. In: J. Pawley (ed.): Handbook of Biological Confocal Microscopy. Plenum Press, New York, pp. $445-458$

Denk, W., Strickler, J. H., Webb, W. W. (1990): Two-photon laser scanning fluorescence microscopy. Science 248, 73-76.

Fan, G. Y., Fujisaki, H., Miyawaki, A., Tsay, R.-K., Tsien, R. Y., Ellisman, M. H. (1999): Video-rate scanning two-photon excitation fluorescence microscopy and ratio imaging with cameleons. Biophys. J. 76, $2412-2420$.

Fujita, K., Nakamura, O., Kaneko, T., Kawata, S., Oyamada, M., Takamatsu, T. (1999): Real-time imaging of two-photon-induced fluorescence with a microlense-array scanner and a regenerative amplifier. J. Microsc. 194, 528 -531.

Fujita, K., Nakamura, N., Kaneko, T., Oyamada, M., Takamatsu, T., Kawata, S. (2000): Confocal multipoint multiphoton excitation microscope with microlens and pinhole arrays. Opt. Commun. 174, $7-12$.

Hell, S. W., Bahlmann, K., Schrader, M., Soini, A., Malak, H., Gryczynski, I., Lakowicz, J. R. (1996): Three-photon excitation in fluorescence microscopy. J. Biomed. Opt. 1, 71-73.

Hell, S. W., Schrader, M., van der Voort, H. T. M. (1997): Far-field fluorescence microscopy with three-dimensional resolution in the $100 \mathrm{~nm}$ range. J. Microsc. 185, $1-5$.

Henkel, A. W., Almers, W. (1996): Fast steps in exocytosis studied by capacitance measurements in endocrine cells. Curr. Opin. Neurobiol. 6, 350-357.

Kim, K. H., Buehler, C., So, P. T. C. (1999): High-speed, two-photon scanning microscope. Appl. Opt. 38, 6004-6009.

Levi, A., Biocca, S., Cattaneo, A., Calissano, P. (1988): The mode of action of nerve growth factor in PC12 cells. Mol. Neurobiol. 2, 201 226.

Manivannan, S., Terakawa, S. (1994): Rapid sprouting of filopodia in nerve terminals of chromaffin cells, PC12 cells, and dorsal root neurons induced by electrical stimulation. J. Neurosci. 14, 5917-5928.

Millot, C., Millot, J. M., Morjani, H., Desplaces, A., Manfait, M. (1997): Characterization of acidic vesicles in multidrug-resistant and sensitive cancer cells by acridine orange staining and confocal microspectrofluorometry. J. Histochem. Cytochem. 45, 1255-1264.

Petit, J. M., Denis-Gay, M., Ratinaud, M. H. (1993): Assessment of fluorochromes for cellular structure and function studies by flow cytometry. Biol. Cell 78, 1-13.

Piston, D. W. (1999): Imaging living cells and tissues by two-photon excitation microscopy. Trends Cell Biol. 9, 66-69.

Plattner, H., Artalejo, A. R., Neher, E. (1997): Ultrastructural organization of bovine chromaffin cell cortex - analysis by cryofixation and morphometry of aspects pertinent to exocytosis. J. Cell Biol. 139, $1709-1717$.

Potter, S. M. (1996): Vital imaging: two photons are better than one. Curr. Biol. 6, 1595-1598.

Sakmann, B., Neher, E. (eds.) (1995): Single-Channel Recording. $2^{\text {nd }}$ edition. Plenum Press, New York.

Smith, C., Moser, T., Xu, T., Neher, E. (1998): Cytosolic $\mathrm{Ca}^{2+}$ acts by two separate pathways to modulate the supply of release-competent vesicles in chromaffin cells. Neuron 20, 1243-1253.

Squirrel, J. M., Wokosin, D. L., White, J. G., Bavister, B. D. (1999): Long-term two-photon fluorescence imaging of mammalian embryos without compromising viability. Nature Biotechnology 17, $763-767$

Steyer, J. A., Almers, W. (1999): Tracking single secretory granules in live chromaffin cells by evanescent-field fluorescence microscopy. Biophys. J. 76, 2262-2271.

Steyer, J. A., Horstmann, H., Almers, W. (1997): Transport, docking and exocytosis of single secretory granules in live chromaffin cells. Nature 388, 474-477.

Stout, A. L., Axelrodt, D. (1989): Evanescent field excitation of fluorescence by epiillumination microscopy. Appl. Optics 28, $5237-5242$

Straub, M., Hell, S. W. (1998a): Multifocal multiphoton microscopy: a fast and efficient tool for 3-D fluorescence imaging. Bioimaging $\mathbf{6}$, $177-185$.

Straub, M., Hell, S. W. (1998b): Fluorescence lifetime three-dimensional microscopy with picosecond precision using a multifocal multiphoton microscope. Appl. Phys. Lett. 73, 1769-1771.

Straub, M., Hell, S. W. (1999): Multifocal multiphoton microscopy as an efficient 4-D imaging method. In: Biomedical Optics: New Concepts in Therapeutic Laser Applications, Novel Biomedical Optical Spectroscopy, Imaging and Diagnostics, Advances in Optical Imaging, Photon Migration and Tissue Optics, OSA Technical Digest (Optical Society of America, Washington DC), p. 373.

Svoboda, K., Denk, W., Kleinfeld, D., Tank, D. W. (1997): In vivo dendritic calcium dynamics in neocortical pyramidal neurons. Nature 385, $161-165$.

Tan, Y. P., Llano, I., Hopt, A., Würriehausen, F., Neher, E. (1999): Fast scanning and efficient photodetection in a simple two-photon microscope. J. Neurosci. Methods 92, 123-135.

Tischler, A. S., Green, L. A. (1976): Establishment of a noradrenergic clonal line of rat adrenal pheochromocytoma cells which respond to nerve growth factor. Proc. Natl. Acad. Sci. USA 73, 2424-2428.

Wokosin, D. L., Centonze, V. E., White, J. G., Hird, S. N., Sepsenwol, S., Malcolm, G. P. A., Maker, G. T., Ferguson, A. I. (1996): Multiphoton excitation imaging with an all-solid-state laser. Proc. SPIE 2678, 38 39.

Yuste, R. and Denk, W. (1995): Dendritic spines as basic function units of neuronal integration. Nature 375, 682-684. 